liche RNS-Änderungen erfolgen: 1. Im Kernsaft der Ei- und Furchungskerne von Cyclops, der in der Interphase keine nachweisbare RNS enthält, sammelt sich in dèr Prophase RNS an, und zwar bereits zu einer Zeit, in der die Kernmembran und z. T. die Nucleolen noch nicht aufgelöst sind. 2. Die RNS des Kernsaftes läßt sich in der intranucleär entstehenden Spindel wieder vorfinden. Wie weit diese RNS-Umsetzungen der Kernteile mit den während der Prophase im Cytoplasma nachweisbaren RNS-Änderungen ${ }^{3}$ in Korrelation stehen, bleibt noch eine offene Frage.

18 H. S ti c h, Chromosoma [1951] (unveröffentlicht).
Neben einer RNS-Ansammlung im Kernsaft des Prophasekernes und der Teilungsspindel konnten in diesen Strukturen auch Polysaccharide nachgewiesen werden ${ }^{18}$. Hiermit sind die in der Mitose ablaufenden Stoffwechselvorgänge zweier Stoffe z. T. aufgedeckt, die mit der Spindelbildung in Verbindung gebracht werden können. Bei einer Zurückführung von Kernanomalien, die nach Bestrahlung oder nach Einwirkung von Mitosegiften auftreten, auf die Störung des Stoffwechsels der Nucleinsäuren oder der Kohlenhydrate, wird man diese extrachromosomalen Prozesse ebenfalls in Erwägung ziehen müssen.

\title{
Vorkommen und Bedeutung von Blastokolinen in fleischigen Früchten
}

\author{
Von Franz Moewus, Liselotte Moewus und Elisabeth Schader \\ Aus dem Max-Planck-Institut für medizinische Forschung, Heidelberg \\ (Z. Naturforschg. 6 b,261-270 [1951]; eingegangen am 10. Mai 1951)
}

\begin{abstract}
Es wird eine Übersicht über die chemische Natur und biologische Bedeutung der Blastokoline gegeben. Als Testobjekte zum Nachweis von Blastokolinen eignet sich ein StandardSaatgut, dessen Keimungsbedingungen bestens bekannt sind (Kresse, Weizen). Zum quantitativen Vergleich ist nicht nur die Feststellung des Keimprozentsatzes, sondern auch der Wurzellänge notwendig. Es wurde die Wirksamkeit von 167 Extrakten aus fleischigen Früchten bestimmt.

Blastokoline sind genuine Stoffe, die die biologische Funktion der Keimungshemmung haben. Nachgewiesen ist ihr Vorhandensein in fleischigen Früchten, Samen, Pollenkörnern und Zygoten.
\end{abstract}

1. Die chemische Natur der Blastokoline Tn seinen Untersuchungen „Über eine keimungshemmende Substanz in fleischigen Früchten “ kommt Kö ckemann ${ }^{1}$ zu folgendem Ergebnis: Preßsäfte von Äpfeln, Birnen, Quitten, Feigen und Tomaten enthalten einen Stoff, der die Keimung von Kressesamen hemmt. Er meint, daß es sich um einen allgemein verbreiteten Stoff handeln müsse, dem er den Namen Blas tokolin ( $\beta \lambda \alpha \sigma \tau \alpha \nu \varepsilon \iota v=$ keimen, $\chi \omega \lambda v \varepsilon \iota v=$ hindern) beilegt. In einer weiteren Arbeit ${ }^{2}$ nimmt er noch einmal „Zur Frage der keimungshemmenden Substanzen in fleischigen Früchten "Stellung. Er bleibt dabei, daß "die Arbeiten von Oppenheimer ${ }^{3}, \mathrm{Fukaki}^{4}$, Reinhar $\mathrm{d}^{5}$ und Köcke$\mathrm{m} a \mathrm{n}{ }^{1}$ die Existenz einer hitzebeständigen, leicht oxydablen, äther- und wasserlöslichen Säure, welche

1 A. Kö c k e m a n n, Ber. dtsch. Bot. Ges. 52, 523 [1934].

2 A. Kö ck e m a n n, Beih. z. Bot. Zbl. A 55, 191 [1936].

3 H. O p p e $\mathrm{n}$ h e i m e r, S.-B. Akad. Wiss. Wien, Abt. I 131, 59, 279 [1922].

4 S. F u k a k i, Bul. Sci. Fak. Terkultura, Kjusu Imp. Univ. Fukuoka, Japanujo 4 [1930]. in reifen Früchten und deren Säften das Auskeimen von Samen verhindert, bewiesen haben“. Allerdings gibt er zu, daß „eine chemische Charakterisierung dieses Stoffes bis heute nicht geglückt ist“.

Im Jahre 1859 isolierte $\mathrm{H}$ of $\mathrm{mann}^{6}$ aus reifen Früchten von Sorbus aucuparia (Vogelbeeren) das rechtsdrehende Sorbinöl (Parasorbinsäure, Hexen-(2)olid-(5.1). Kuhn und Jerchel ${ }^{7}$ bewiesen durch Abbau und Synthese die Konstitution dieser Verbindung. Sollte sie das von $\mathrm{Köckemann}$ vermutete Blastokolin fleischiger Früchte sein? Tatsächlich stellte es sich heraus, daß die Keimung von Kressesamen noch in Verdünnungen von 1:1000 gehemmt wurde ${ }^{8}$. Bestünde die Annahme von Köckemann zu Recht, so müßte die Parasorbinsäure in allen fleischigen Früchten für die Keimungshemmung verantwortlich sein.

5 A. W. R e in h a r d, Planta 20, 792 [1933].

6 A. W. H of $\mathrm{m}$ a $\mathrm{n} n$, Liebigs Ann. Chem. 210, 129 [1859].

7 R. K u h n u. D. J e r che l, Ber. dtsch. chem. Ges. 76, 413 [1943].

8 R. Kuhn, D. Jerchel, F. Moewus, E. F. Möller u. H. Le t $\mathrm{t} r$ é, Naturwiss. 31, 468 [1943] 
Wir begannen im Jahre 1939 gemeinsam mit F. W e y g a nd Tomatenmark zu untersuchen, um die chemische Natur des Blastokolins aufzuklären ${ }^{9}$. Es wurde damals eine hochwirksame Fraktion erhalten. Nach Weygand ${ }^{10}$ hatte aber das TomatenBlastokolin nicht die Eigenschaften des Hexenolids aus Vogelbeeren. A. M. A k kerman und Veldstra ${ }^{11}$ isolierten später aus Tomaten zwei Säuren, die für die keimungshemmende Wirkung des Fruchtfleisches verantwortlich gemacht werden: Kaffeesäure (3.4-Dioxyzimtsäure) und Ferulasäure (3-Methoxy-4-oxy-zimtsäure). Damit war bewiesen, daß es nicht ein allgemein verbreitetes Blastokolin gibt, sondern daß in fleischigen Früchten Stoffe enthalten sind, die chemisch verschiedenen Klassen angehören können, denen aber eine keimungshemmende Wirkung gemeinsam ist.

\section{Die biologische Bedeutung der Blastokoline}

Der Beweis für die biologische Bedeutung dieser genuinen Stoffe für die Pflanze ist die Austestung mit den arteigenen Samen. Wir haben Preßsäfte von Tomaten mit frischen, aus den Früchten isolierten Samen zu testen versucht. Jedoch setzte während der langen Keimzeit ein starker Pilzbefall ein, so daß die Versuche nicht ausgewertet werden konnten. Eine Pilzinfektion ändert die physiologischen Bedingungen im Keimbett, weil Hemmstoffe und Wuchsstoffe von dem Pilz gebildet und ausgeschieden werden können. Versuche mit Samen von Sorbus aucuparia, die im arteigenen Fruchtextrakt zur Keimung ausgelegt wurden, scheiterten ebenfalls an der auftretenden Pilzinfektion. Die Samen keimten selbst in den Wasserkontrollen nicht aus. R e in h a r d ${ }^{5}$ hat 1933 die Wirkung von Tomatensaft auf Tomatensamen geprüft. Wie aus seiner Arbeit hervorgeht, hemmt dieser nicht nur die Keimung, sondern auch das Wachstum der Keimlingswurzeln. In diesem Fall dürfte die biologische Bedeutung der Stoffe, die im Fruchtfleisch vorhanden sind und die die Keimung der reifen, wasserreichen Samen innerhalb der Frucht verhindern, wohl als bewiesen gelten.

$\mathrm{Pa}$ e ch ${ }^{12}$ untersuchte das Verhalten von Kakaosamen verschiedenen Reifegrades, die er mit und ohne Schleimhüllen zur Keimung brachte. Reife

9 F. M o ew u s, FIAT Review of German Science. Biochemie 2, 185 [1948].

10 F. W e y g a n d, Die Chemie 56, 236 [1943]. Diskussionsbemerkung.

11 H. V e ld stra, Rec. trav. chim. Pays-Bas. 66, 411 [1947].
Samen keimten sowohl mit als auch ohne Schleimhülle aus. Daraus wurde gefolgert, daß in den Hüllen der reifen Samen keine spezifischen Keimhemmungsstoffe vorhanden sind. Der Versuch, unreife Samen von ihren Hüllen zu befreien oder gründlich auszuwaschen und dann auf ihre Keimfähigkeit zu prüfen, wurde leider nicht gemacht. Daß die Schleimhüllen dennoch Stoffe enthalten, welche einen Einfluß auf die Keimung haben, wurde mit Kresse nachgewiesen. Es wurden Extrakte aus Schleimhüllen hergestellt und darin Kressesamen zur Keimung gebracht. Es ließ sich feststellen, daß in reifen und überreifen Schleimextrakten weniger Hemmsubstanzen enthalten sind. Die gebotenen Konzentrationen vermögen nur noch eine hemmende Wirkung auf das Wachstum der Kressewurzel auszuüben, nicht aber die Keimung zu verhindern. $\mathrm{P}$ a e c $\mathrm{h}$ sieht in dem verschiedenen Verhalten von Kressesamen und Kakaosamen gegenüber Hemmstoffen reifer Hüllen den Beweis, daß es sich um keine spezifischen Keimungshemmstoffe handeln könne. Sie erfüllen nicht die biologische Funktion der Keimungshemmung gegenüber dem arteigenen Samen. Das Auskeimen von Samen innerhalb überreifer Früchte, das $\mathrm{Pa}$ e ch ebenfalls als Beweis anführt, ist nicht überraschend, da „es immer nur dann beobachtet wurde, wenn das Perikarp von Schimmelpilzen befallen war". Tab. 7 zeigt, daß es einige unter den geprüften Fruchtextrakten gibt, bei denen mit Kresse eine Abnahme des Hemmstoffgehaltes während der Reife festgestellt wurde. Zumeist ist jedoch eine Zunahme gefunden worden. Die Prüfung, wie sich Extrakte verschiedener Reifegrade gegenüber arteigenen Samen verhalten, ist von uns nicht durchgeführt worden. Aber auch $\mathrm{Pa}$ e $\mathrm{ch}$ hat nicht den Versuch gemacht, reife Samen in Extrakten unreifer Hüllen zum Keimen zu bringen. Die von $\mathrm{Pa}$ e ch erhaltenen Ergebnisse können die Befunde von R e in h a rd nicht entkräften, so daß die biologische Bedeutung der Blastokoline fleischiger Früchte nicht bezweifelt werden kann ${ }^{13}$.

\section{Testobjekte}

zum Nachweis von Blastokolinen

Wie eben beschrieben wurde, ist der Nachweis spezifischer Keimungshemmstoffe in einem Fruchtextrakt mit arteigenem Samen nur schwer zu erbrin-

12 K. P a e c h, Z. Naturforschg. 4 b, 46 [1947].

13 Die Zahl der Kakao-Früchte (5!) und Samen (etwa 100 !), mit denen $\mathrm{P}$ a e $\mathrm{c} \mathrm{h}$ experimentiert hat, ist so gering, daß aus den erhaltenen Ergebnissen keine entscheidenden Schlußfolgerungen gezogen werden können. 
gen, da die Samen oft wochen- oder monatelang im Keimbett ruhen, bevor sie zu keimen beginnen, auch wenn ihnen reines Wasser geboten wird. Ein Standard-Saatgut, dessen Keimungsbedingungen bestens bekannt sind, und das in Wasser in kurzer Zeit zu annähernd $100 \%$ keimt, ist also die direkte Voraussetzung für vergleichende Versuchsreihen. Seit Wi es $\mathrm{n}$ e $\mathrm{r}^{14}$ werden dazu Kressesamen (Lepidium sativum) verwendet. Even ari und Mitarbeiter ${ }^{15}$, die ausgedehnte Versuche auf diesem Gebiete ausgeführt haben; nehmen mit gleichem Erfolg Weizen. In der Evenari-Schule wurde festgestellt, daß sich verschiedene Samensorten gegen einen Fruchtextrakt verschieden empfindlich erweisen. Zum Beispiel hemmt Tomatensaft bei einer Verdünnung von 1:25 die Keimung von Kressesamen zu $100 \%$. Bei Weizen muß für den gleichen Effekt eine Verdünnung von 1: 4 angewendet werden. Fruchtextrakt der Zuckerrübe, $1: 4$ verdünnt, hemmt Amaranthus-Samen zu $100 \%$, Kressesamen zu $96 \%$, während er auf Samen von Ipomoea purpurea keinerlei hemmenden Einfluß hat. Kressesamen ist also sehr empfindlich. Er ist bestens als Testobjekt zum Nachweis keimungshemmender Stoffe geeignet. Man muß sich nur im Klaren sein, daß mit der leicht $\mathrm{zu}$ beeinflussenden Kresse noch geringe Mengen von Hemmstoffen nachweisbar sind, die für den arteigenen Samen bereits unwirksam sein könnten, wie es bei Kakaosamen der Fall zu sein scheint. Eine bei Kresse erreichte Keimhemmung ist nicht ohne weiteres gleichzusetzen mit dem Nachweis eines artspezifischen, biologisch wirksamen Blastokolins. Jeder Test ist ein Modellversuch, bei dem die natürlichen Bedingungen nur teilweise erfüllt sind. Es ist aber unmöglich, größere Serien vergleichender Untersuchungen auszuführen, wenn Versuchsbedingungen und Versuchsobjekte nicht bis zum höchstmöglichen Grade standardisiert sind. Eine erste Voraussetzung für die Austestung von Fruchtextrakten ist eine kurze Versuchszeit, um Veränderungen im Keimbett durch Bakterien- und Pilzbefall möglichst einzuschränken. Die schnellkeimende Kresse ist deshalb Weizen und anderen Samen vorzuziehen.

\section{Quantitative Bestimmung des Blastokolin-Gehaltes}

Um graduelle Unterschiede in der Wirksamkeit verschiedener Preßsäfte zu finden, müssen Verdünnungsreihen hergestellt werden, da unverdünnte

14 J. W i e s ne r, S.-B. Akad. Wiss. Wien, Abt. I 103 [1894].

15 M. Even a ri , Botanic. Rev. 15, 153 [1949].
Preßsäfte fast stets die Keimung von Kresse völlig unterdrücken. Die Verdünnungsreihen können nach zweierlei Gesichtspunkten bereitet werden. 1. Man versucht, für jeden Preßsaft die Grenzkonzentration zu finden, bei der die hemmende Wirkung gerade aufhört. Dies läßt sich mit einiger Sicherheit feststellen, wenn kleinste Verdünnungsstufen geprüft werden $(1: 1,1: 2,1: 3$ usw.). In der Versuchspraxis erweist sich diese Methode als umständlich. Die Ergebnisse der einzelnen Versuche lassen sich nur schwer reproduzieren und sichern. 2. Man wählt größere Intervalle, aber stets die gleichen (unverdünnt, $1: 10$, 1:100). Gleichzeitig wird eine Wasserkontrolle angesetzt. Dann werden die gekeimten Samen jeder Versuchsschale ausgezählt und prozentual auf die Wasserkontrolle bezogen, die gleich 100 gesetzt wird. Ein solches Versuchsergebnis besteht also in 3 Zahlen. Die Intervalle sind meist $\mathrm{zu}$ weit voneinander entfernt, so daß die Grenzkonzentration nicht erfaßt wird. Bei unseren Versuchen (vgl. Tab. 1-7) zeigte oft die 1:10-Verdünnung keine Keimhemmung mehr, oder einen Wert, der dicht bei der Wasserkontrolle oder bei "ungekeimt“ liegt.

Die Evenari-Schule ${ }^{15}$, die kleinere Verdünnungsintervalle anwendet $(1: 2,1: 4,1: 8,1: 16$ usw.) findet graduell abgestufte Keimprozente bei den einzelnen Lösungen. Wir zogen trotzdem die Anwendung größerer Verdünnungsstufen vor, und zwar aus folgenden Gründen. Es wird immer wieder der Einwand erhoben, daß die Keimhemmung in fleischigen Früchten nicht durch einen spezifischen Keimungshemmstoff bedingt sei, sondern daß andere Faktoren im Fruchtfleisch dafür verantwortlich zu machen wären 16: der hohe Zuckergehalt, hohe Acidität, Gerbstoffgehalt usw. Wir haben die einzelnen Faktoren eingehend untersucht ${ }^{9}$ - ebenso auch die Even ariSchule - und fanden, daß sie zwar in natürlichen Preßsäften durch Summation eine keimungshemmende Wirkung entfalten können. In einer $1: 10$-Verdün-

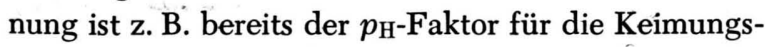
hemmung unwesentlich geworden, wie aus den Tab. 1-7 überall hervorgeht. Speziell für Tomatensaft ist von $\mathrm{K}$ o n i s ${ }^{17}$ nachgewiesen worden, daß der osmotische Druck die Keimungshemmung nicht bedingt, ebensowenig die Wasserstoffionenkonzentration. Pflanzliche Preßsäfte enthalten in der Regel neben Hemmstoffen auch Wuchsstoffe. Heteroauxin hemmt in konzentrierteren Lösungen natürlich auch die Keimung von Kressesamen, wie eine im Jahre 1944

16 W. T e t j u r e w, Planta 32, 211 [1941/42].

17 E. Kon is, Palest. J. Bot., Jerusalem, Ser. 2, 6 [1940]. 


\begin{tabular}{|c|l|r|r|r|r|r|c|c|}
\hline \multirow{2}{*}{ Nr. } & Sorte & \multicolumn{6}{|c|}{ Keimungshemmung in \% } & \multicolumn{3}{|c|}{ Wachsiumsiemming in \% } & $p_{\mathrm{H}}$ des \\
& & 1 & $1: 10$ & $1: 100$ & 1 & $1: 10$ & $1: 100$ & Preßsaftes \\
\hline 1 & Große Fleisch & 100 & 100 & 0 & 100 & 100 & 61 & 4,5 \\
2 & Radio & 100 & 100 & 2 & 100 & 100 & 23 & 4,2 \\
3 & Vater Rhein & 100 & 27 & 0 & 100 & 89 & 14 & 4,2 \\
4 & Dänischer Export & 100 & 2 & 2 & 100 & 85 & 32 & 4,2 \\
5 & Undefatigable & 100 & 7 & 8 & 100 & 44 & 26 & 4,1 \\
6 & Heterosis & 100 & 6 & 0 & 100 & 69 & 10 & 4,3 \\
7 & Immun & 100 & 2 & +2 & 100 & 56 & 22 & 4,4 \\
8 & Niederer Busch & 2 & 4 & 3 & 94 & 90 & 29 & 4,3 \\
& X Blondköpfchen & 7 & 1 & 0 & 83 & 20 & 14 & 4,2 \\
9 & Lucullus & 7 & 52 & +1 & 93 & 93 & 17 & 4,2 \\
\hline
\end{tabular}

Tab. 1. Die Wirkung von Preßsäften von zehn verschie lenen Tomatensorten im Keimungs- und Wachstumstest. Angegeben sind die prozentualen Hemmungen gegenüber der Wasserkontrolle im unverdünnten Preßsaft (1) und in den 1:10- sowie 1:100-Verdünnungen. + bedeutet Förderung. Reife Früchte.

\begin{tabular}{|c|c|c|c|c|c|c|c|c|}
\hline \multirow{2}{*}{ Nr. } & \multirow{2}{*}{ Art bzw. Sorte } & \multicolumn{3}{|c|}{ Keimungshemmung in $\%$} & \multicolumn{3}{|c|}{ Wachstumshemmung in $\%$} & \multirow{2}{*}{$\begin{array}{c}p_{\mathrm{H}} \text { des } \\
\text { Preßsaftes }\end{array}$} \\
\hline & & 1 & $1: 10$ & $1: 100$ & 1 & $1: 10$ & $1: 100$ & \\
\hline 1 & Rosa canina & 100 & 100 & 5 & 100 & 100 & 17 & 4,0 \\
\hline 2 & Rosa rugosa & 100 & 27 & 1 & 100 & 92 & 5 & 4,1 \\
\hline 3 & Rubus idaeus & 100 & 56 & +1 & 100 & 91 & 22 & 3,3 \\
\hline 4 & Rubus idaeus & 100 & 100 & 2 & 100 & 100 & 9 & 3,2 \\
\hline 5 & Rubus laciniatus & 100 & 100 & +4 & 100 & 100 & +4 & 3,0 \\
\hline 6 & Rubus nitidus & 100 & 100 & +5 & 100 & 100 & +5 & 3,7 \\
\hline 7 & Fragaria vesca & 100 & 100 & 6 & 100 & 100 & 8 & 3,5 \\
\hline 8 & Fragaria vesca & 100 & 100 & 1 & 100 & 100 & 5 & 3,5 \\
\hline 9 & $\begin{array}{l}\text { Monatserdbeere } \\
\text { Gartenerdbeeren: }\end{array}$ & 100 & $\cdot 100$ & 5 & 100 & 100 & 41 & 3,8 \\
\hline 10 & Königin Luise & 100 & 100 & 6 & 100 & 100 & 24 & 3,5 \\
\hline 11 & Oberschlesien & 100 & 100 & 3 & 100 & 100 & 29 & 3,5 \\
\hline 12 & Madame Moutot & 100 & 100 & 3 & 100 & 100 & 40 & 3,5 \\
\hline & & & $000=$ & & & $000=$ & & \\
\hline
\end{tabular}

Tab. 2. Die keimungs- und wachstumshemmende Wirkung von Preßsäften verschiedener Rosoideenfrüchte. Die zwei Bestimmungen bei 3 und 4 sowie 7 und 8 liegen 4 Wochen auseinander.

durchgeführte Austestung ergab. Bei $10^{-6} \mathrm{~g} / \mathrm{ccm}$ ist die Keimung nur noch zu $14 \%$ gehemmt, ab $10^{-7} \mathrm{~g} / \mathrm{ccm}$ wird sie nicht mehr beeinflußt. Im unverdünnten Preßsaft sind die Wuchsstoffkonzentrationen jedoch wesentlich geringer, so daß die Wuchsstoffe die Keimung im Preßsaft nicht hemmen.

Geht man also von der Voraussetzung aus, daß es spezifische, hochwirksame Keimungshemmstoffe gibt, dann ist es vorteilhaft, mit solchen Verdünnungsstufen $\mathrm{zu}$ arbeiten, bei denen man sich bereits jenseits der Wirksamkeitsgrenze der ebenfalls keimhemmenden Faktoren befindet. Betrachten wir die Ergebnisse, die mit Preßsäften von 5 Sorbus-Arten erhalten wurden:

\begin{tabular}{|c|c|c|c|c|}
\hline & \multicolumn{3}{|c|}{ Keimungshemmung in $\%$} & $p_{\mathrm{H}} \operatorname{des}$ \\
\hline Verdünnungsstufe: & 1 & $1: 10$ & $1: 100$ & Preßsaftes \\
\hline Sorbus moravia & 100 & 100 & 9 & 3,2 \\
\hline Sorbus torminalis & 100 & 100 & 2 & 4,1 \\
\hline Sorbus aucuparia & 100 & 34 & 0 & 3,4 \\
\hline Sorbus Aria & 100 & 20 & 1 & 4,2 \\
\hline Sorbus domestica & 100 & 3 & +4 & 3,2 \\
\hline
\end{tabular}

Die beiden Arten mit dem niedrigsten $p_{\mathrm{H}}$-Wert $(3,2)$ hemmen in einem Fall bei $1: 10$ noch zu $100 \%$, im andern Fall nicht mehr (3\%). Daraus geht eindeutig hervor, daß die Hemmung nicht von der Acidität des natürlichen Saftes bedingt sein kann. Auch die anderen Werte sind nicht durch $p_{\mathrm{H}}$-bedingte Keimungshemmung zu erklären. Aus den $1: 10$-Verdünnungen kann man schließen, daß die zwei ersten Arten und die letzte sich bestimmt im Gehalt ihrer Hemmstoffe unterscheiden. Bei den beiden mittleren Arten ist dies aus der 1:10-Verdünnung nicht ohne weiteres zu ersehen. Betrachtet man die 1:100-Verdünnung, dann kann bei allen 5 Arten nichts über eine unterschiedliche Wirksamkeit in diesem Bereich ausgesagt werden. Mit der Bestimmung der Keimprozente können allgemein orientierende, vergleichende Aussagen über den Hemmstoffgehalt gemacht werden.

Eine weitere Charakterisierung der einzelnen Sorbus-Arten läßt sich durchführen, wenn man das 


\begin{tabular}{|c|c|c|c|c|c|c|c|c|}
\hline \multirow{2}{*}{ Nr. } & \multirow{2}{*}{ Art } & \multicolumn{3}{|c|}{ Keimungshemmung in $\%$} & \multicolumn{3}{|c|}{ Wachstumshemmung in $\%$} & \multirow{2}{*}{$\begin{array}{c}p_{\mathrm{H}} \text { des } \\
\text { Preßsaftes }\end{array}$} \\
\hline & & 1 & $1: 10$ & $1: 100$ & 1 & $1: 10$ & $1: 100$ & \\
\hline 1 & Sorbus moravica & 100 & 100 & 9 & 100 & 100 & 49 & 3,2 \\
\hline 2 & Sorbus torminalis & 100 & 100 & 2 & 100 & 100 & 25 & 4,1 \\
\hline 3 & Sorbus aucuparia & 100 & 34 & 0 & 100 & 93 & 13 & 3,4 \\
\hline 4 & Sorbus Aria & 100 & 20 & 1 & 100 & 91 & 15 & 4,2 \\
\hline 5 & Sorbus domestica & 100 & 3 & +4 & 100 & 90 & 9 & 3,2 \\
\hline 6 & Pirus salicifolia & 100 & 55 & +1 & 100 & 92 & 5 & 3,3 \\
\hline 7 & Pirus amygdaliformis & 100 & 31 & 1 & 100 & 92 & 24 & 3,9 \\
\hline 8 & Pirus betulifolia & 100 & 18 & 3 & 100 & 92 & 16 & 3,8 \\
\hline 9 & Pirus elaeagnifolia & 100 & 6 & 4 & 100 & 54 & +3 & 4,0 \\
\hline 10 & Pirus cordata & 100 & 2 & 2 & 100 & 44 & +7 & 3,5 \\
\hline 11 & Pirus communis subsp. nivalis & 100 & $+\overline{3}$ & $+\overline{1}$ & 100 & 22 & +3 & 3,8 \\
\hline 12 & $\begin{array}{l}\text { Pirus communis subsp. sativa } \\
\text { „Alexander“ }\end{array}$ & 100 & 31 & +3 & 100 & 92 & 6 & 4,5 \\
\hline 13 & Malus arbutifolia & 100 & 42 & 1 & 100 & 92 & +1 & 4,0 \\
\hline 14 & Malus Scheideckeri & 100 & 14 & 0 & 100 & 92 & +3 & 3,3 \\
\hline 15 & Malus floribunda & 100 & 3 & 2 & 100 & 89 & +1 & 3,9 \\
\hline 16 & Malus cerasifera & 100 & +2 & 3 & 100 & 61 & 3 & 3,0 \\
\hline 17 & Malus orthrocarpa & 100 & 7 & 2 & 100 & 55 & 0 & 4,0 \\
\hline 18 & Malus baccata & 100 & 3 & 0 & 100 & 45 & 9 & 3,0 \\
\hline 19 & Malus pumila & 100 & 1 & +3 & 100 & 34 & 3 & 4,3 \\
\hline 20 & Malus Graham & 100 & +4 & +2 & 100 & 6 & 0 & 3,2 \\
\hline 21 & $\begin{array}{l}\text { Malus communis } \\
\text { „Kasseler Renette” }\end{array}$ & 100 & 3 & +2 & 100 & 45 & 2 & 3,3 \\
\hline
\end{tabular}

Tab. 3. Die keimungs- und wachstumshemmende Wirkung von Preßsäften aus Früchten der Pomoideen-Gattungen Sorbus, Pirus und Malus.

\begin{tabular}{|c|c|c|c|c|c|c|c|c|}
\hline \multirow{2}{*}{ Nr. } & \multirow{2}{*}{ Art bzw. Sorte } & \multicolumn{3}{|c|}{ Keimungshemmung in $\%$} & \multicolumn{3}{|c|}{ Wachstumshemmung in $\%$} & \multirow{2}{*}{$\begin{array}{c}p_{\mathrm{H}} \text { des } \\
\text { Preßsaftes }\end{array}$} \\
\hline & & 1 & $1: 10$ & $1: 100$ & 1 & $1: 10$ & $1: 100$ & \\
\hline 1 & Prunus cerasus (Schattenmorelle) & 100 & 100 & 9 & 100 & 100 & 34 & 3,5 \\
\hline 2 & P. avium (rote Herz) & 100 & 100 & 11 & 100 & 100 & 50 & 3,5 \\
\hline 3 & P. avium (schwarze Knorpel) & 100 & +4 & 1 & 100 & 68 & 12 & 3,8 \\
\hline 4 & P. armeniaca (Aprikose) & 100 & 100 & 8 & 100 & 100 & 40 & 3,5 \\
\hline 5 & P.domestica (Mirabelle) & 100 & 100 & +5 & 100 & 100 & 22 & 3,8 \\
\hline 6 & P. domestica (Reineclaude) & 100 & 100 & 1 & 100 & 100 & 10 & 3,2 \\
\hline 7 & P.domestica (Herbstzwetschge) & 100 & 55 & 7 & 100 & 91 & 30 & 3,8 \\
\hline 8 & P. domestica (Lützelsachsener) & 100 & 100 & 2 & 100 & 100 & 54 & 3,5 \\
\hline 9 & P. domestica (Eierpflaume) & 100 & 100 & 30 & 100 & 100 & 79 & 3,5 \\
\hline 10 & P. domestica (Eisenkehler) & 100 & 100 & 33 & 100 & 100 & 70 & 3,5 \\
\hline
\end{tabular}

Tab. 4. Die keimungs- und wachstumshemmende Wirkung von Preßsäften aus verschiedenen Prunus-Früchten.

Verhalten der Wurzeln von Kresse-Keimlingen bei den einzelnen Verdünnungsstufen beachtet. Es zeigt sich, daß das Wurzelwachstum viel empfindlicher auf Hemmstoffe reagiert (vgl. Tab. 3). Jetzt findet man bei den $1: 100$-Verdünnungen noch eine deutliche Beeinflussung. Die Unterschiede im Hemmstoffgehalt der 5 Arten treten jetzt besser hervor. Die erste Art enthält etwa doppelt so viel wie die zweite und etwa 5-mal so viel wie die letzte Art. Bei allen untersuchten Preßsäften wurde deshalb neben der prozentualen Keimungshemmung auch die prozentuale Wachstumshemmung gegenüber der nicht beeinflußten Wasserkontrolle bestimmt, so daß ein Versuchsergebnis sich in 6 Zahlen ausdrückt. In keinem
Fall wurden Preßsäfte gefunden, die nur die Keimung, nicht aber das Wurzelwachstum hemmen. Andererseits wurden Säfte gefunden, die zwar das Wurzelwachstum hemmen, die Keimung aber nur wenig beeinflussen. Dies war aber nur bei einigen reifen Früchten der Fall (vgl. Tab. 7). Diesen beiden Bei, spielen wäre noch die Kakaofrucht zuzurechnen.

Mit Hilfe des eben beschriebenen Kresse-Testes, der eine Verbindung von Keimungs-Test und Wachstums-Test darstellt, wurden in den Jahren 1943-46 mehr als 500 verschiedene pflanzliche Extrakte auf ihre keimungs- und wachstumshemmende Wirkung untersucht. In allen Fällen wurde eine Hemmwirkung angetroffen, die bei den einzelnen Pflanzenarten je- 


\begin{tabular}{|c|c|c|c|c|c|c|c|c|c|}
\hline \multicolumn{2}{|c|}{ Berberis } & $\begin{array}{l}\text { Keimungs- } \\
\text { hemmung } \\
\text { in } \%\end{array}$ & $\begin{array}{l}\text { Wachstums- } \\
\text { hemmung } \\
\text { in } \%\end{array}$ & $p_{\mathbf{H}}$ & \multicolumn{2}{|c|}{ Berberis } & $\begin{array}{l}\text { Keimungs- } \\
\text { hemmung } \\
\text { in } \%\end{array}$ & $\begin{array}{l}\text { Wachstums- } \\
\text { hemmung } \\
\text { in } \%\end{array}$ & $p_{\mathrm{H}}$ \\
\hline Dealbata & $\begin{array}{l}1 \\
1: 10 \\
1: 100\end{array}$ & $\begin{array}{r}100 \\
28 \\
+2\end{array}$ & $\begin{array}{r}100 \\
89 \\
23\end{array}$ & 3,0 & Spathulata & $\begin{array}{l}1: 1,5 \\
1: 15 \\
1: 150\end{array}$ & $\begin{array}{r}100 \\
10 \\
+4\end{array}$ & $\begin{array}{r}100 \\
88 \\
9\end{array}$ & 2,9 \\
\hline Polyantha & $\begin{array}{l}1 \\
1: 10 \\
1: 100\end{array}$ & $\begin{array}{r}100 \\
100 \\
0\end{array}$ & $\begin{array}{r}100 \\
100 \\
0\end{array}$ & 2,9 & Provincialis & $\begin{array}{l}1: 10 \\
1: 100 \\
1: 1000\end{array}$ & $\begin{array}{r}100 \\
+7 \\
+5\end{array}$ & $\begin{array}{r}100 \\
61 \\
+9\end{array}$ & 3,4 \\
\hline Vulgaris & $\begin{array}{l}1 \\
1: 10 \\
1: 100\end{array}$ & $\begin{array}{r}100 \\
5 \\
6\end{array}$ & $\begin{array}{r}100 \\
89 \\
3\end{array}$ & 3,9 & Hookeri & $\begin{array}{l}1: 10 \\
1: 100 \\
1: 1000\end{array}$ & $\begin{array}{r}100 \\
1 \\
+2\end{array}$ & $\begin{array}{r}100 \\
79 \\
+1\end{array}$ & 3,3 \\
\hline Vernae & $\begin{array}{l}1: \quad 1,17 \\
1: 11,7 \\
1: 117\end{array}$ & $\begin{array}{r}100 \\
27 \\
0\end{array}$ & $\begin{array}{r}100 \\
87 \\
0\end{array}$ & 2,6 & Edworthiana & $\begin{array}{l}1: 10 \\
1: 100 \\
1: 1000\end{array}$ & $\begin{array}{r}100 \\
18 \\
1\end{array}$ & $\begin{array}{r}100 \\
88 \\
16\end{array}$ & 3,0 \\
\hline Crataegina & $\begin{array}{l}1: \quad 1,17 \\
1: 11,7 \\
1: 117\end{array}$ & $\begin{array}{r}100 \\
12 \\
+4\end{array}$ & $\begin{array}{r}100 \\
86 \\
39\end{array}$ & 2,9 & & & & & \\
\hline
\end{tabular}

Tab. 5. Die keimungs- und wachstumshemmende Wirkung von Preßsäften aus Früchten verschiedener Berberis-Arten. Die $p_{\mathrm{H}}$-Werte der Preßsäfte sind stets unverdünnt bestimmt worden.

doch verschieden war. Im folgenden sollen die mit fleischigen Früchten erhaltenen Ergebnisse in Tabellenform zusammengestellt und kurz diskutiert werden. Besonders eingehend wurden Vertreter der Familien Solanaceen, Rosaceen und Berberidaceen untersucht.

\section{Versuchsergebnisse}

a) Sol a n a e e n

Im Jahre 1944 waren wir in der Lage, 25 verschiedene Tomatensorten zu untersuchen, die uns von der Versuchsund Forschungsanstalt für Wein-, Obst- und Gartenbau in Geisenheim (Rhein) zur Verfügung gestellt wurden (Tab. 1). Nr. 1-7 stellt eine Reihe abnehmender Wirksamkeit dar. Auffallend ist das Verhalten von Nr. 8 und 9: Die unverdünnten Preßsäfte beeinflussen nicht die Keimung, wohl aber das Wurzelwachstum sehr stark. Ähnlich verhält sich Nr. 10. Die Sorten „verbesserte Zucker“ und „racemosum“ ähneln Nr. 1 und 2, „Frühbote“, „Kondine Red“, „Blondköpfchen“ Nr. 4, „Standard“, „Sieger“, „Riverside“, „Hellfrucht“, „Rheinlands Ruhm“, „Erste Ernte“, „gelbe Delikateß“ Nr. 5 und 6. Die Ergebnisse zeigen, daß in allen Preßsäften Hemmstoffe vorhanden sind, die die Keimung beeinflussen, noch empfindlicher aber das Wurzelwachstum. Durch diese Versuche sind wesentliche Sortenunterschiede erfaßt worden, während der Nachweis derartiger Hemmstoffe schon früher mehrfach erbracht worden ist 18 .

b) Ros a ce e $n$

Aus der Gruppe der Rosoideen wurden die Gattungen Rosa, Fragaria und Rubus untersucht (Tab. 2). ErdbeerExtrakte sind sehr wirksam. Wie die Sorten „Königin

18 I. Ju el, Dansk Bot. Ark. 12, No. 4 [1946]; P. La r sen, Planta 30, 160 [1939]; vgl. auch Even ari 15, Reinh a r d 5 .
Luise“ und "Oberschlesien“ verhalten sich auch „Deutsches Memelland“ und "Hilgenstein“. Besonders viel Hemmstoffe enthält die Sorte „Madame Moutot“. T e t j u r e w 16 vertrat die Ansicht, daß der Zuckergehalt vieler Früchte die keimungshemmende Ursache sei. Nach B e y th i e $\mathbf{n} 19$ beträgt der durchschnittliche Zuckergehalt von Erdbeeren 6\%. Keimungsversuche mit Kressesamen in Gegenwart verschieden konzentrierter Saccharose-Lösungen ergaben aber, daß eine 10-proz. Lösung die Keimung nicht, das Wachstum schwach beeinflußt, während eine 6-proz. Lösung weder Keimung noch Wachstum hemmt.

Aus der Gruppe der Pomoideen wurden Versuche mit den 3 Gattungen Sorbus, Pirus und Malus angestellt (Tab. 3). Das Material stammte größtenteils aus dem Heidelberger botanischen Garten. Vergleicht man die 3 Gattungen, dann ergibt sich, daß die Vertreter der Gattung Sorbus mehr Hemmstoffe enthalten als die der beiden anderen Gattungen. Bei den Arten jeder Gattung läßt sich eine Reihe abnehmender Wirksamkeit der Preßsäfte aufstellen. Die Kulturformen (Nr. 12 u. 21) nehmen keine Sonderstellung ein. Das Blastokolin der Vogelbeeren, die Parasorbinsäure, hemmt nicht nur die Keimung von Kressesamen und das Wachstum von Kressewurzeln 8, sondern es beeinflußt auch zahlreiche andere Wachstumsprozesse, u. a. das Austreiben von Kartoffelknollen 20 und die Bildung sowie das Wachstum der Rhizoide bei Marchantia polymorpha 21 .

Wie aus Tab. 4 hervorgeht, sind die Preßsäfte von Prunus-Früchten reich an keimungs- und wachstumshemmenden Stoffen. Denn die 1:10-Verdünnungen der Preßsäfte unterdrücken meist noch die Keimung der Kressesamen vollständig. Über die chemische Natur der

19 A. B e y thi e n, Handb. d. Lebensmittelchemie 5, 501 [1938].

20 F. M o e w u s u. E. S c h a d e r, Z. Naturforschg. 6 b, 112 [1951].

21 F. Moe wus u. E. S ch a der, Beitr. Biol. Pflanzen, im Druck. 


\begin{tabular}{|c|c|c|c|c|c|c|c|c|}
\hline \multirow{2}{*}{ Nr. } & \multirow{2}{*}{ Art } & \multicolumn{3}{|c|}{ Keimungshemmung in $\%$} & \multicolumn{3}{|c|}{ Wachstumshemmung in $\%$} & \multirow{2}{*}{$\begin{array}{c}p_{\mathrm{H}} \text { des } \\
\text { Preßsaftes }\end{array}$} \\
\hline & & 1 & $1: 10$ & $1: 100$ & 1 & $1: 10$ & $1: 100$ & \\
\hline 1 & Morus alba & 100 & 100 & 2 & 100 & 100 & 14 & 5,6 \\
\hline 2 & Ribes rubrum „rot” & 100 & 83 & +4 & 100 & 83 & 9 & 2,6 \\
\hline $\overrightarrow{3}$ & Ribes rubrum "weiß" & 100 & 100 & 5 & 100 & 100 & 15 & 3,2 \\
\hline 4 & Ribes nigrum & 100 & 100 & +1 & 100 & 100 & 15 & 2,9 \\
\hline 5 & Ribes grossularia & 100 & 100 & +3 & 100 & 100 & 9 & 3,2 \\
\hline 6 & Hippophae rhamnoides & 100 & 4 & +4 & 100 & 81 & 3 & 2,6 \\
\hline 7 & Vitis vinifera „Riesling” & 100 & 6 & 0 & 100 & 81 & 0 & 3,8 \\
\hline 8 & Vitis vinifera „Müller-Thurgau” & 100 & 49 & 1 & 100 & 91 & 25 & 3,8 \\
\hline 9 & Viburnum Opulus & 100 & 74 & 2 & 100 & 93 & 8 & 5,4 \\
\hline 10 & Sambucus nigra & 100 & 100 & +5 & 100 & 100 & 23 & 4,2 \\
\hline 11 & Symphoricarpus racemosus & 100 & 4 & +3 & 100 & 64 & +7 & 5,1 \\
\hline 12 & Cucurbita Pepo & 100 & 0 & +2 & 100 & 81 & 17 & 5,4 \\
\hline 13 & Cucumis sativus & 45 & $\mathbf{0}$ & $+\overline{2}$ & 91 & +10 & +7 & 5,1 \\
\hline 14 & Taxus baccata & 100 & +1 & 0 & 100 & 8 & +4 & 6,0 \\
\hline 15 & Juniperus communis & 100 & 8 & 3 & 100 & 46 & +7 & $5,1 \times$ \\
\hline
\end{tabular}

Tab. 6. Die keimungs- und wachstumshemmende Wirkung von Preßsäften aus Früchten verschiedener Arten. $x=$ der Extrakt aus Wacholderbeeren wurde dadurch gewonnen, daß $10 \mathrm{~g}$ Frucht zerrieben und mit $25 \mathrm{ccm}$ Wasser versetzt wurden; 1,5 Stdn. später wurde filtriert (= Lösung 1).

Hemmstoffe bei Prunus ist nichts bekannt. Von anderer Seite 22 sind bei Pfirsichen Keimungshemmstoffe nachgewiesen worden.

c) Berberidace en

In Tab. 5 sind die Wirkungen der Preßsäfte aus Früchten von 9 Berberis-Arten angeführt. Da von einigen Arten nur geringe Mengen Früchte zur Verfügung standen, konnten die Original-Preßsäfte nicht getestet werden. Die an letzter Stelle stehenden Arten, B. Hookeri und B. Edworthiana, sind besonders reich an Hemmstoffen. Der Vergleich von B. dealbata und B. Edworthiana zeigt wieder, daß die verschiedene Wirkung der Preßsäfte nicht auf die Wasserstoffionenkonzentration zurückzuführen ist.

d) Sonstige fleischige Früchte

Tab. 6 zeigt die Wirkungen von Preßsäften aus anderen fleischigen Früchten. Von anderer Seite sind früher schon bei Vitis vinifera ${ }^{15}$, Cucumis sativus 23 und Juniperus communis 24 Hemmstoffe nachgewiesen worden.

e) Vergleich von unreifen und reifen Früchten

Im Laufe der Untersuchungen mit fleischigen Früchten konnten bei einigen Arten auch Früchte verschiedenen Reifegrades verglichen werden. Bei den in Tab. 7 zuerst angeführten Arten ist der Hemmstoffgehalt von reifen Früchten höher als derjenige in unreifen Früchten. Dies kommt sowohl in der Keimungshemmung als auch in der Wachstumshemmung deutlich zum Ausdruck, oder nur in der Wachstumshemmung. Bei Sorbus aucuparia wurden 3 verschiedene Reifegrade untersucht. Es ergibt sich eine Zunahme des Hemmstoffgehaltes während der Reife. Dagegen bleibt der Wuchsstoffgehalt während der Reife konstant, wie spätere Untersuchungen gezeigt haben 25 . Bei einigen Arten ist der Hemmstoffgehalt in unreifen

22 D. H. S cott, J. G. W a u g h u. F. P. Cullim a n, Amer. Soc. Hort. Sci. 40, 283 [1942].

23 E. K a u f m a n n, Planta 33, 516 [1943].
Früchten größer als in reifen. Besonders deutlich kommt dies bei Symphoricarpus zum Ausdruck. Ob dieses abweichende Verhalten nicht nur vorgetäuscht ist, könnte erst entschieden werden, wenn als Bezugsgröße das Trockengewicht gewählt wird und wenn das Verhalten arteigenen Samens untersucht wird.

f) Zus a m menf as sung

Von der Jerusalemer Schule und von uns sind während der letzten Jahre in den fleischigen Früchten vieler Familien Blastokoline festgestellt worden. In Tab. 8 ist eine Übersicht, nach Familien geordnet, gegeben. Unsere Ergebnisse gründen sich auf die Untersuchung von $167 \mathrm{Ex}$ trakten in 565 Einzelversuchen mit insgesamt 56500 Kressesamen bzw. Kressekeimlingen. Dieser einfache Kresse-Test erlaubt es, quantitative Bestimmungen des BlastokolinGehaltes in pflanzlichen Extrakten und Preßsäften vorzunehmen. Zugleich mit der Keimungshemmung wurde eine hemmende Beeinflussung des Wurzelwachstums des Keimlings festgestellt. Die Wurzel reagiert in der Regel etwa 10-mal empfindlicher auf die in den Preßsäften enthaltenen Hemmstoffe.

\section{Was sind Blastokoline?}

Auf Grund unserer Untersuchungen lassen sich folgende Aussagen über die Eigenschaften der Blastokoline machen. Blastokoline sind stets genuine Stoffe in fleischigen Früchten, denen die biologische Funktion der Keimungshemmung des arteigenen Samens zugesprochen werden muß. Die biologische Keimungshemmung von Tomatenmark auf Tomatensamen ist von $\mathrm{Re} \mathrm{inhar \textrm {d } ^ { 5 }}$ bewiesen. Es ist noch zu untersuchen, ob die von Akkerman und

24 S. B. U $11 \mathrm{man}$, zitiert bei Evenari15 (Ph. D. Thesis, Jerusalem 1940).

25 F. M o e w u s, Biol. Zbl. 68, 58 [1949]. 


\begin{tabular}{|c|c|c|c|c|c|c|c|c|c|c|}
\hline \multirow{2}{*}{$\mathrm{Nr}$. } & \multirow{2}{*}{ Art } & & \multicolumn{3}{|c|}{ Keimungshemmung in $\%$} & \multicolumn{3}{|c|}{ Wachstumshemmung in $\%$} & \multirow{2}{*}{$p_{\mathrm{H}}$} & \multirow{2}{*}{ Bemerkungen } \\
\hline & & & 1 & $1: 10$ & $1: 100$ & 1 & $1: 10$ & $1: 100$ & & \\
\hline \multirow[t]{2}{*}{1} & Rubus idaeus & $\mathbf{u}$ & 100 & 50 & 5 & 100 & 73 & 6 & 2,9 & (langfrüchtig) \\
\hline & & $\mathrm{r}$ & 100 & 100 & 5 & 100 & 100 & 20 & 3,5 & \\
\hline \multirow[t]{2}{*}{2} & Rubus idaeus & $\mathrm{u}$ & 100 & 35 & 2 & 100 & 82 & 0 & 2,9 & (rundfrüchtig) \\
\hline & & $\mathbf{r}$ & 100 & 100 & 8 & 100 & 100 & 12 & 3,5 & \\
\hline \multirow[t]{3}{*}{3} & Brombeere & $\mathbf{u}$ & 40 & 20 & 6 & 92 & 92 & 16 & 2,6 & (grün) \\
\hline & & $\mathrm{u}$ & 62 & 7 & +1 & 92 & 92 & 12 & 2,6 & (hellrot) \\
\hline & & $\mathrm{r}$ & 100 & 38 & 5 & 100 & 92 & 26 & 3,0 & \\
\hline \multirow[t]{2}{*}{4} & Morus nigra & $\mathrm{u}$ & 100 & 100 & 2 & 100 & 100 & +6 & 4,0 & \\
\hline & & $\mathrm{r}$ & 100 & 100 & +1 & 100 & 100 & 16 & 4,3 & \\
\hline \multirow[t]{2}{*}{5} & Sambucus nigra & $\mathrm{u}$ & 100 & 100 & +5 & 100 & 100 & 11 & 3,5 & \\
\hline & & $\mathrm{r}$ & 100 & 100 & +5 & 100 & 100 & 23 & 4,2 & \\
\hline \multirow[t]{2}{*}{6} & Juniperus communis & $\mathrm{u}$ & 100 & 5 & +2 & 100 & 2 & +12 & 5,1 & $($ je $10 \mathrm{~g}+25$ \\
\hline & & $\mathrm{r}$ & 100 & 8 & 3 & 100 & 46 & +7 & 5,1 & ccm Wasser) \\
\hline \multirow[t]{3}{*}{7} & Sorbus aucuparia & $\mathrm{u}$ & 100 & +1 & 0 & 100 & 29 & 3 & 3,0 & (grün) \\
\hline & & $\mathrm{u}$ & 100 & 11 & 0 & 100 & 80 & 0 & 3,2 & (gelbrot) \\
\hline & Tomaten: & $\mathbf{r}$ & 100 & 34 & 0 & 100 & 93 & 13 & 3,4 & (rot) \\
\hline \multirow[t]{2}{*}{$\varepsilon$} & „Bonner Beste” & $\mathrm{u}$ & 100 & 5 & 3 & 100 & 39 & +10 & 4,0 & \\
\hline & & $\mathbf{r}$ & 100 & 2 & 4 & 100 & 56 & 30 & 4,2 & \\
\hline \multirow[t]{2}{*}{9} & "gelbe Delikatess" & $\mathrm{u}$ & 100 & 6 & 3 & 101 & 27 & 16 & 4,2 & \\
\hline & & $\mathbf{r}$ & 100 & 3 & 4 & 100 & 70 & 20 & 4,2 & \\
\hline \multirow[t]{2}{*}{10} & „Kondine Red” & $\mathrm{u}$ & 100 & +2 & 0 & 100 & 40 & 14 & 4,1 & \\
\hline & & $\mathbf{r}$ & 100 & 10 & 0 & 100 & 80 & 21 & 4,1 & \\
\hline \multirow[t]{2}{*}{11} & „Blondköpfchen” & $\mathbf{u}$ & 100 & 6 & +1 & 100 & 71 & 16 & 4,2 & $\cdot$ \\
\hline & & $\mathbf{r}$ & 100 & +2 & +1 & 100 & 90 & 31 & 4,2 & \\
\hline \multirow[t]{2}{*}{12} & „Frühbote” & $\mathbf{u}$ & 48 & 13 & 4 & 89 & 79 & 39 & 4,1 & \\
\hline & & $\mathbf{r}$ & 100 & 82 & 5 & 100 & 78 & 28 & 4,2 & \\
\hline \multirow[t]{2}{*}{13} & „verbesserte Zucker” & $\mathrm{u}$ & 100 & 4 & +4 & 100 & 92 & 9 & 3,9 & \\
\hline & & $\mathbf{r}$ & 100 & 7 & 2 & 100 & 94 & 21 & 3,9 & \\
\hline \multirow[t]{2}{*}{14} & „racemosum“ & $\mathbf{u}$ & 100 & 8 & 1 & 100 & 94 & 4 & 4,0 & \\
\hline & & $\mathbf{r}$ & 100 & 11 & 1 & 100 & 94 & 16 & 4,0 & \\
\hline \multirow[t]{2}{*}{15} & Malus coronaria & $\mathrm{u}$ & 100 & 5 & 0 & 100 & 86 & 16 & 3,6 & \\
\hline & & $\mathbf{r}$ & 100 & +2 & 4 & 100 & 56 & +2 & 2,9 & \\
\hline 16 & Viburnum Opulus & $\mathbf{u}$ & 100 & 100 & +4 & 100 & 100 & 6 & 4,5 & \\
\hline & & $\mathbf{r}$ & 100 & 74 & 2 & 100 & 93 & 8 & 5,4 & \\
\hline 17 & Symphoricarpus & $\mathbf{u}$ & 100 & 100 & 3 & 100 & 100 & 85 & 4,5 & (grün) \\
\hline & racemosus & $\mathbf{u}$ & 100 & 41 & +2 & 100 & 88 & 36 & 5,1 & (hellgrün) \\
\hline & & $\mathbf{r}$ & 100 & 4 & +3 & 100 & 64 & +7 & 5,1 & (weiß) \\
\hline 18 & Cucumis sativus & $\mathbf{u}$ & 93 & 1 & 4 & 84 & 20 & 5 & 5,4 & \\
\hline & & $\mathbf{r}$ & 45 & 0 & +2 & 91 & +10 & +7 & 5,1 & \\
\hline 19 & Tomate„erste Ernte” & $\mathbf{u}$ & 100 & 2 & 0 & 100 & 72 & 59 & 4,2 & \\
\hline & & $\mathbf{r}$ & 100 & 8 & 2 & 100 & 67 & 24 & 4,0 & \\
\hline 20 & rote Kirschtomate & $\mathrm{u}$ & 100 & 35 & 4 & 100 & 80 & 15 & 4,2 & \\
\hline 21 & Taxus baccata & $\mathrm{u}$ & 100 & $\begin{array}{r}52 \\
2\end{array}$ & $\begin{array}{l}+1 \\
+2\end{array}$ & $\begin{array}{r}93 \\
100\end{array}$ & $\begin{array}{l}93 \\
69\end{array}$ & $\begin{array}{l}16 \\
16\end{array}$ & $\begin{array}{l}4,2 \\
5,1\end{array}$ & (unreif: $1: 2$ \\
\hline & & $\mathrm{r}$ & 100 & +1 & 0 & 100 & 8 & +4 & 6,0 & $1: 20,1: 200)$ \\
\hline
\end{tabular}

Tab. 7. Die keimungs- und wachstumshemmende Wirkung von Preßsäften aus Früchten verschiedenen Reifegrades.

Veldstra ${ }^{11}$ isolierte Kaffeesäure und Ferulasäure auf Tomatensamen hochspezifisch keimhemmend wirken. Für Sorbus aucuparia ist zu vermuten, daß die von $\mathrm{Kuhn}$ und J er ch e ${ }^{7}$ isolierte Parasorbinsäure das Blastokolin darstellt, obwohl auch hier der biologische Nachweis mit arteigenen Samen noch nicht vorliegt.

26 H. F u n ke, Beih. z. Bot. Zbl. A 59, 235 [1939].

27 A. M. Ozorio de Almeida, M. D. Goulart, M. I elpo u. A. Viera P into, Rev. brasil. Biol. 1, 345 [1941].

28 E. K o n i s, Thesis. Hebrew Univ. Jerusalem [1938]; Palest. J. Bot. Jerusalem Ser. 2, 6 [1940].
Werden Blastokoline durch ihre physiologische Fähigkeit der Keimungshemmung charakterisiert, dann sind als echte Blastokoline bisher noch zwei weitere Fälle bekannt.

1. Bei Forsythia intermedia wurde aus Pollenkörnern der Langgriffler-Blüten Rutin, aus Pollen-

29 E. K o n i s, Palest. J. Bot. Jerusalem, Ser. 4 [1947].

30 M. S t o u t u. B. T o l m a n, J. agric. Res. 63, 687 [1941].

31 R. S r o e lov, Palest. J. Bot. Jerusalem, Ser. 2, 33 [1940].

32 R. K u h n u. I. L öw, Chem. Ber. 82, 474 [1949]. 


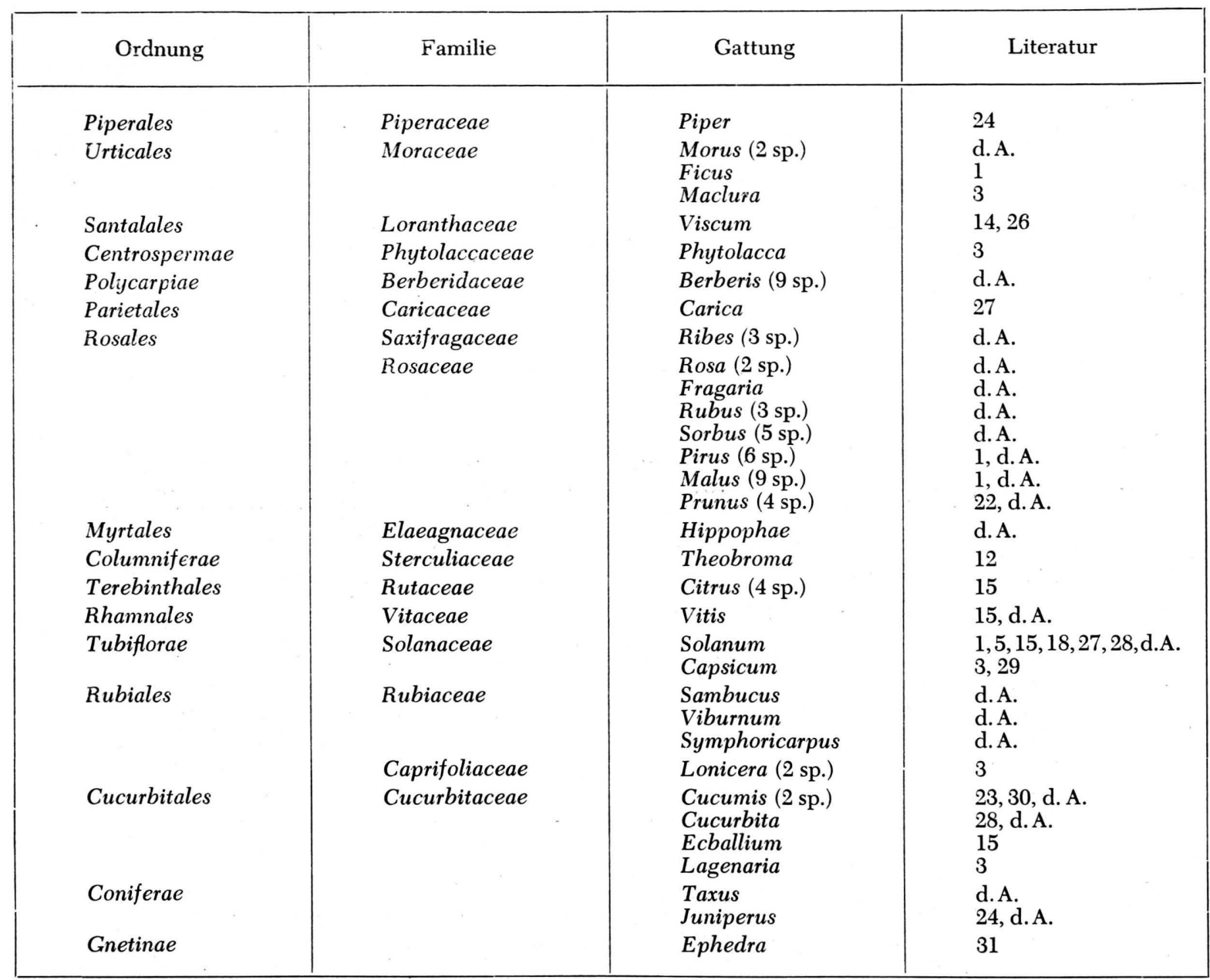

Tab. 8. Das Vorkommen von Blastokolinen in Extrakten aus fleischigen Früchten (die mit „d. A.“ bezeichneten wurden von uns untersucht).

körnern der Kurzgriffler-Blüten Quercitrin isoliert ${ }^{32}$. Beide Flavonole unterdrücken noch in einer Verdünnung von $1: 10^{6} \mathrm{~g} / \mathrm{ccm}$ die Bildung der Pollenschläuche bei keimfähigen Pollen ${ }^{3}$. Ihre biologische Funktion besteht darin, die Keimung von Pollen auf Narben des gleichen Blütentyps zu verhindern.

2. In Extrakten von Zygoten der Grünalge Chlamydomonas eugametos ist ein keimungshemmender Stoff enthalten, der die Keimung (Aufteilung in 4 Gonen) von Zygoten hemmt, die durch Auswaschen keimfähig gemacht worden sind ${ }^{34}$. Die stärkste Keimungshemmung wurde mit cis-Zimtsäure erzielt, die

38 F. M o ew u s, Biol. Zbl. 69, 181 [1950].

34 F. M o ew u s, Z. Naturforschg. 5 b, 196 [1950].

35 F. Mo ew u s u. B. B a n e r j e e, Z. Naturforschg. 6 b, 270 [1951].

\& R. M a r x, H. B a y e r l e u. E. Marx, Biochem. Z. 319, 378 [1949]. im Gegensatz $\mathrm{zu}$ anderen isomeren Verbindungen hochwirksam ist und bei einer Verdünnung von $1: 800000000$ die Keimung noch zu 50\% hemmt ${ }^{35}$. Die Isolierung dieses Zygoten-Blastokolins steht allerdings noch aus.

Auch mit den Pollen- und Zygoten-Blastokolinen wurde im Kressewurzel-Test eine Hemmung des Wurzelwachstums erzielt. Nur müssen für diesen Effekt höhere Dosen angewendet werden.

Auch in Samen sind Blastokoline nachgewiesen worden. In Tab. 1, bei Eve n a r i ${ }^{15}$, finden sich zahlreiche Angaben, u. a. Apium graveolens, Brassica nigra, Coffea arabica, Lactuca sativa, Phacelia tanacetifolia, Pisum, Trifolium, zahlreiche Gramineen.

Im Jahre 1949 veröffentlichten $\mathrm{M}$ a r x und Mitarbeiter $^{36}$ eine Arbeit „Úber die Blastokolin- und Rhizakolin-Wirkung einiger Cumarinderivate ... . 
Die Autoren erweitern den Begriff Blastokolin auf alle Verbindungen, welche die Keimung bei einer Verdünnung von mindestens $1: 10000$ deutlich herabsetzen. Dabei ist die Grundvoraussetzung, daß die Blastokoline genuine Stoffe sind, völlig vernachlässigt. Es genügt nicht, Blastokoline durch ihre Wirksamkeit bei $1: 10000 \mathrm{zu}$ charakterisieren. Auch die Unterscheidung besonderer Rhizakoline, die nur eine Hemmung des Wurzelwachstums bedingen, ist unnötig. Denn ob Sprosse oder Wurzeln gehemmt werden, ist abhängig von der Dosierung. Jedes Rhizakolin hemmt bei einer genügend hohen Dosis sowohl die Keimung als auch das Sproßwachstum.

Der Begriff Blastokolin soilte nur dann gebraucht werden, wenn die biologische Funktion in Extrakten mit arteigenen Samen (oder Pollen, Zygoten) nachgewiesen ist. Sonst spricht man besser allgemein von Keimungshemmstoff (germination inhibitors ${ }^{15}$ ) oder von wachstumshemmenden Stoffen (growth inhibitors).

\title{
Über die Wirkung von cis-Zimtsäure und einigen isomeren Verbindungen auf Chlamydomonas-Zygoten
}

\author{
Von Franz Moewus und Basudev Banerjee* \\ Aus dem Max-Planck-Institut für medizinische Forschung, Institut für Chemie, Heidelberg \\ (Z. Naturforschg. 6 b, 270-273 [1951]; eingegangen am 11. Mai 1951)
}

Im Keimungstest mit Zygoten der blastikos-Rasse von Chlamydomonas eugametos haben Zimtaldehyd, $o$-Cumarsäure und $\beta$ - $d$-Glucosido-o-cumarsäure nur eine geringe keimungshemmende Wirkung. Cumarin ist etwa 100-mal wirksamer, trans-Zimtsäure etwa 10-mal aktiver als Cumarin. Die Wirkung der cis-Zimtsäure übertrifft die der trans-Form um fast das 100-fache. Die 3 allotropen Modifikationen der cis-Zimtsäure (Schmp. $42^{\circ}, 58^{\circ}, 68^{\circ}$ ) verhalten sich physiologisch gleichartig. Cumarin ergibt bei $10^{-8} \mathrm{~g} / \mathrm{ccm}$, cis-Zimtsäure bei $10^{-11}$ und $10^{-12} \mathrm{~g} / \mathrm{ccm}$ eine statistisch gesicherte Keimungsförderung gegenüber der Kontrolle.

$\mathrm{D}$ ie Zygoten der Grünalge Chlamydomonas eugametos enthalten einen Keimungshemmstoff (Blastokolin) ${ }^{1}$. Ihre Keimung setzt erst dann ein, wenn die Konzentration dieses Stoffes innerhalb der Zelle herabgesetzt wird. Bei der Rasse blastikos läßt sich die Keimung auslösen, indem man die Zygoten aus dem Kulturmedium, in welchem sie sich seit der Entstehung befanden, in frische Nährlösung überträgt. Dadurch entsteht ein Diffusionsgefälle. Der Blastokolingehalt im Zellinnern sinkt. Nach nochmaligem Wechsel sind die Zygoten dann zu mehr als 85\% keimfähig. Diese ausgewaschenen Zygoten wurden als Testmaterial zum Nachweis von Stoffen verwendet, die eine keimungshemmende Wirkung ausüben.

Da über die chemische Natur des Blastokolins nichts bekannt war, wurde zunächst die Wirkung einiger organischer Säuren geprüft. Dabei hat sich ergeben, daß trans-Zimtsäure die Zygotenkeimung auffallend stark hemmt ${ }^{1}$ : bei einer Konzentration von $10^{-7} \mathrm{~g} / \mathrm{ccm}$

1 F. M o ew u s, Z. Naturforschg. 5 b, 196 [1950].

2 J. B. Koepfli, K. V. Thimann, F. W. Went, J. biol. Chemistry 122, 763 [1937/38]; H. Veldstra, Enzymologie [Den Haag] 11, 97 [1943/45].

3 G. E b e r t, Liebigs Ann. Chem. 226, 347 [1884]. wird die Keimung noch um etwa 50\% gegenüber der Kontrolle unterdrückt. Auch Cumarin, das Lacton der Cumarinsäure (cis-2-Oxy-zimtsäure) hat sich als sehr wirksam erwiesen. In verschiedenen pflanzlichen Wuchsstofftesten ${ }^{2}$ hatte sich gezeigt, daß cis-Zimtsäure im Gegensatz zur trans-Form wachstumsfördernd wirkt. Es war daher wichtig zu wissen, welche Wirkung cis-Zimtsäure und andere isomere Verbindungen auf Chlamydomonas-Zygoten ausüben.

Folgende Verbindungen wurden im Keimungs-Test mit Chlamydomonas-Zygoten vergleichend untersucht:

1. Zimtaldehyd.

2. o-Cumarsäure (trans-2-Oxy-zimtsäure). Diese erhielten wir durch alkalische Hydrolyse von Cumarin mit Natriumalkoholat nach der Methode von Ebert ${ }^{3}$.

3. $\beta$ - $d$-Glucosido-o-cumarsäure. Sie wurde dargestellt durch Kondensation von Helicin mit Malonsäure in Gegenwart von Pyridin und einigen Tropfen Anilin nach $\mathrm{Helferich}$ und $\mathrm{Lutz}$ man ${ }^{4}$.

* Senior Research Fellow of Bose Institute Calcutta, working as a Govt. of India Scholar.

4 B. He lf e ri c h u. H. L u t z m a n n, Liebigs Ann. Chem. 537, 11 [1939]. 